\title{
Medición de la incidencia de eventos adversos atribuidos a la atención sanitaria en un Departamento de Pediatría de un hospital universitario
}

\author{
Measurement of the incidence of care-associated adverse events at \\ the Department of Pediatrics of a teaching hospital
}

Dra. Ana Fajreldines ${ }^{a}$, Dr. Eduardo Schnitzler ${ }^{t b}$, Dr. Silvio Torres ${ }^{c}$,

Dr. Néstor Panattieri y Dr. Marcelo Pellizzaria

\section{RESUMEN}

Introducción. Los eventos adversos pueden ser detectados por diversas herramientas de pesquisa. En la población pediátrica, la Pediatric Global Trigger Tool busca gatillos en la historia clínica para detectar daño asociado al cuidado, tanto prevenible como no prevenible.

Objetivo. Medirlaincidenciadeeventos adversos en elDepartamento de Pediatría utilizando dicha herramienta. Conocer los tipos de eventos y las variables asociadas.

Población y métodos. Estudio de incidencia, de corte longitudinal.

Resultados. Se estudiaron 318 pacientes; media de edad: 2,99 años (rango: 0-17); porcentaje de mujeres: 164 (el 51,57 \%). Se halló un $11 \%$ de eventos por cada 100 admisiones, 15,5 eventos cada 1000 días-paciente. Los eventos más frecuentes fueron asociados a medicación: el $48,57 \%$ ( $\mathrm{n}=17)$; a bacteriología (infecciones): el $42,85 \%(\mathrm{n}=15)$; y a cuidados: el $8,57 \%(\mathrm{n}=3)$. Diecinueve eventos fueron leves (el 54,28\%), 14 (el $40 \%$ ) prolongaron la hospitalización (moderados) y 2 (el 5,71 \%) requirieron soporte vital (graves). Se hallaron 168 gatillos, 0,53 gatillos por paciente, 74,4 gatillos cada 1000 días-paciente y 4,8 gatillos por cada evento adverso. En el análisis de regresión logística multivariada, las variables asociadas a la aparición de los eventos adversos fueron medicamentos de alto riesgo, sexo femenino, peso, número de transferencias dentro del hospital y estancia superior a 5 días. Conclusiones. La utilización de la Pediatric Global Trigger Tool permiteidentificar eventos adversos en pacientes pediátricos hospitalizados y ayudar a encauzar acciones de mejora, de acuerdo con las variables asociadas.

Palabras clave: Global Trigger Tool, eventos adversos, niño hospitalizado.

http:/ / dx.doi.org/10.5546/ aap.2019.e106

Texto completo en inglés:

http:/ / dx.doi.org/10.5546/ aap.2019.eng.e106

Financiamiento:

Ninguno.

Conflicto de intereses: Ninguno que declarar.

Recibido: 2-2-2018

Aceptado: 26-11-2018
Cómo citar: Fajreldines A, Schnitzler E, Torres S, Panattieri N, Pellizzari M. Medición de la incidencia de eventos adversos atribuidos a la atención sanitaria en un Departamento de Pediatría de un hospital universitario. Arch Argent Pediatr 2019;117(2):e106-e109.

\section{INTRODUCCIÓN}

Los eventos adversos en los hospitales continúan siendo un problema no resuelto y, en consecuencia, afectan gravemente la seguridad de los pacientes. La pediatría ofrece alternativas de tratamiento a situaciones complejas que desafían la confiabilidad de los procesos de atención. ${ }^{1}$ Baker et al., reportan una incidencia de eventos adversos en internación de adultos del 7,5\%. ${ }^{2}$ En los hospitales pediátricos, la incidencia alcanza un $9,2 \%$, y son más frecuentes en centros académicos que en hospitales de comunidad. ${ }^{3}$ De los métodos de pesquisa, el reporte voluntario es el más utilizado, aunque subestima, en forma importante, la real incidencia. ${ }^{4}$

La Global Trigger Tool (GTT), desarrollada por el Institute for Healthcare Improvement, ${ }^{5}$ utiliza la revisión de historias clínicas para identificar gatillos que pueden asociarse con eventos adversos y ha demostrado tener más sensibilidad que el sistema de reporte voluntario. ${ }^{6}$

La GTT fue adaptada para la población pediátrica $(\text { GTTP })^{7}$ y ha sido aplicada en hospitales pediátricos. Kirkendall y col., ${ }^{8}$ hallaron una incidencia de 1,7 gatillos por paciente, un evento adverso cada 4,6 gatillos y 36,7 eventos cada 100 admisiones. En nuestro país, Davenport ${ }^{9}$ halló una tasa de 26 eventos adversos cada 100 admisiones en una muestra de 200 pacientes con esta misma metodología. 
Los objetivos del presente trabajo fueron estimar la incidencia de eventos adversos en pacientes pediátricos hospitalizados, conocer los tipos más frecuentes de eventos adversos y las variables asociadas a estos, utilizando la herramienta de gatillos (GTTP).

\section{POBLACIÓN Y MÉTODOS}

Estudio de incidencia, de corte longitudinal, que incluyó a pacientes de 0-17 años inclusive, con, al menos, 48 h de hospitalización. El estudio se efectuó en un hospital universitario de alta complejidad con historia clínica electrónica. El período analizado fue de julio de 2015 a julio de 2016.

Se realizó un muestreo de pacientes egresados mediante la aleatorización de números emitidos por Excel (Windows 7). El tamaño muestral se calculó sobre la fórmula de proporciones con un universo conocido (2000 egresos anuales), error alfa de 0,05, intervalo de confianza (IC) del $95 \%$, precisión del $3 \%$. La proporción de eventos adversos fue del $40 \%$. El número de egresos pediátricos fue el $20 \%$ del total de egresos. Los días-paciente mensuales fueron entre 1100 y 1300.

Se revisaron todos los componentes de la historia clínica: evoluciones, medicamentos, laboratorios, dietas, interconsultas, protocolos quirúrgicos y epicrisis, que alcanzaron una completitud del 80-85\% según un monitoreo interno acorde a los estándares de Joint Commission International (JCI).

En 2014, se efectuó una prueba piloto con la GTTP, sobre 50 casos, y se obtuvo un kappa de 0,78 entre observadores (IC $95 \%$ : 0,11-0,97).

Las medidas de resultado fueron las recomendadas por la GTTP: tasa de eventos cada 100 admisiones, tasa de eventos cada 1000 díaspaciente y proporción de admisiones con, al menos, 1 evento adverso.

La escala de daño usada fue la recomendada por la herramienta. ${ }^{10}$ Las categorías fueron daño E: requería intervención (leve); daño F: prolongaba la hospitalización (moderado); daño $\mathrm{G}$ (grave): permanente; daño H (grave): requería soporte vital; daño I: provocaba o contribuía a la muerte (grave).

Los pacientes con más de 2 transferencias fueron aquellos que tuvieron pasajes desde y hacia áreas de hospitalización o donde recibieron tratamientos o procedimientos: Cirugía, Hemodinamia, Cuidados Críticos, etc.

Se consideró medicación de alto riesgo a drogas oncológicas, electrolitos concentrados, insulina, entre otros, según la lista de recomendaciones de este grupo de medicamentos del Institute for Safe Medication Practices (ISMP). ${ }^{11}$

Para el análisis estadístico, se usó el programa SPSS $19, \mathrm{IBM}^{\circledR}$. El test $\chi^{2}$ para variables dicotómicas y el test de análisis de la varianza (analysis of variance; ANOVA, por sus siglas en inglés), para variables continuas. Se realizó una regresión binaria logística para analizar la asociación de variables.

Se contó con la aprobación del Comité Institucional de Evaluación (CIE).

\section{RESULTADOS}

Se revisaron 318 historias clínicas. La media de edad fue 2,99 años (rango: 0-17); desvío estándar (DE): 6,64; mujeres: el 51,57 \% (164); varones: el 48,43\% (154). Los días-paciente fueron 2257. Las áreas de hospitalización fueron Sala Pediátrica General: 145 (el 45,6\%); Nursery: 82 (el 25,8\%); Unidad de Terapia Intensiva: 65 (el 20,4\%); Unidad de Cuidados Neonatales: 26 (el 8,2\%). El total de eventos adversos hallados fueron 35 , que correspondieron al $11 \%(10,2-12,6)$ cada 100 admisiones, 15,5 cada 1000 días-paciente y el 8,17 \% (IC $95 \%$ : 77,4-82,4) de admisiones con, al menos, un evento adverso.

Los eventos cada 100 admisiones por área fueron el $0 \%$ para Nursery, $7,69(6,5-8,3)$ para Neonatología, 12,41 $(11,1-13,5)$ para Sala Pediátrica General y 18,29 $(16,9-18,8)$ para Terapia Intensiva.

Los eventos más frecuentes fueron los asociados a medicación: el 48,6\% ( $\mathrm{n}=17)$; a microbiología (infecciones): el 42,8 \% ( $\mathrm{n}=15)$; y a cuidados: el $86 \%(n=3)$. No se constataron muertes. Según la clasificación de daño, los eventos fueron 19 (el 54,28\%) de tipo leve, $14(\mathrm{el} 40 \%)$ prolongaron la hospitalización, y 2 (el 5,71\%) graves, que necesitaron soporte vital. Fueron hallados 168 gatillos, 0,53 gatillos por paciente, 74,4 gatillos cada 1000 díaspaciente y 4,8 gatillos cada 1 evento adverso. Los gatillos más frecuentes fueron los de cuidados: 98 (el 58,3\%); medicación: 45 (el 26,78 \%); y microbiología: 25 (el 14,88\%), coincidente con los daños encontrados. Los eventos adversos más frecuentes fueron desaturación y complicación de algún procedimiento, los de medicación (vómitos) y los de microbiología (infecciones), con hemocultivo positivo. Las incidencias por área fueron Sala Pediátrica General: 145 (el 45,60\%); Nursery: 82 (el 25,79\%); Unidad de Terapia 
Intensiva: 65 (el 20,44\%); Unidad de Cuidados Neonatales: 26 (el 8,17\%). Según el área de hospitalización, fueron del $0 \%$ para Nursery, del 7,69\% para Neonatología, del 12,41\% para Sala Pediátrica General y del 18,29 \% para Terapia Intensiva.

En Cuidados Intensivos, se produjeron, proporcionalmente, más eventos cada 100 admisiones que en Internación General: $18,29$ vs. 12,41 ( $p=0,05)$.

Los días de estancia prolongados se contabilizaron a partir del día de la aparición del evento. La media de estancia prolongada fue 3,14 días.

Las variables asociadas a la aparición de los eventos adversos fueron medicamentos de alto riesgo, peso, sexo femenino, número de transferencias dentro del Hospital y estancia superior a 5 días (Tabla 1).

\section{DISCUSIÓN}

Los estudios que analizaron este tema, como los de Stockwell et al., ${ }^{12}$ y Kirkendall et al., ${ }^{8}$ hallaron tasas de 40 y 36,7 eventos adversos por cada 100 admisiones, respectivamente. Kirkerdall et al., ${ }^{8}$ refirieron una mayor frecuencia de eventos relacionados con cirugía (el $39 \%$ ), mientras que, en nuestro estudio, la medicación (el $54 \%$ ) fue la principal causa. En nuestro Hospital, el proceso de medicación no utilizaba sistemas expertos de soporte, sino un sistema electrónico con barreras de seguridad manuales.

El estudio de Takata et al., ${ }^{13}$ mostró eventos adversos a medicamentos más numerosos que otras publicaciones. Su estudio analizó más de 900 pacientes y halló 11,11 eventos adversos a medicamentos por cada 100 pacientes, concordante, en términos de relevancia, con nuestros datos. Stroupe et al., ${ }^{14}$ en un estudio canadiense que utilizó el Global Assessment of Pediatric Patient Safety (GAPPS), ${ }^{15}$ hallaron tasas menores que las nuestras y que las de los autores mencionados en este estudio sobre 100 casos: el $4,87 \%$ de eventos adversos.
Davenport et al., ${ }^{9}$ encontraron 26 eventos cada 100 admisiones y 1,4 gatillos por paciente con índices de daño y de gatillos mayores que los de nuestro estudio $(0,53$ gatillos por paciente y 11 eventos cada 100 admisiones en nuestro estudio). Quizá la diferencia pueda explicarse por la inclusión de pacientes de baja complejidad de la Sala Pediátrica General y de Nursery. Sin embargo, la metodología requiere que todo paciente hospitalizado participe del muestreo y que este sea representativo de los egresos totales.

La prolongación de la estancia por daño asociado al cuidado fue hallada también por autores como Palacios Barahona et al., ${ }^{16}$ Kaushal et al., ${ }^{17}$ y Hug et al. ${ }^{18}$

\section{CONCLUSIONES}

A pesar de ser una de las primeras experiencias de uso de la GTTP en nuestro país, y de estar dentro de la literatura variada que hay sobre el tema y los resultados amplios en términos de daño a pacientes pediátricos, se considera que la herramienta abre la oportunidad para rediseñar procesos y mejorar la seguridad del paciente.

La presencia de medicamentos de alto riesgo, peso, sexo femenino, número de transferencias dentro del Hospital y estancia superior a 5 días fueron variables asociadas a eventos adversos.

\section{REFERENCIAS}

1. Porter ME. What is Value in Health Care? N Engl J Med. 2010; 363(26):2477-81.

2. Baker GR, Norton PG, Flintoft V, Blais R, et al. The Canadian Adverse Events Study: the incidence of adverse events among hospital patients in Canada. CMAJ. 2004; 170(11):1678-86.

3. Matlow AG, Baker GR, Flintoft V, Cochrane D, et al. Adverse events among children in Canadian hospitals: the Canadian Pediatric Adverse Events Study. CMAJ. 2012; 184(13):E709-18.

4. AHRQQIResearch Version 5.0, Pediatric Quality Indicators \#19, Technical Specifications. Pediatric Patient Safety for Selected Indicators. [Consulta: 3 de diciembre de 2017]. Disponible en: www.qualityindicators.ahrq.gov/ Downloads / Modules / PDI/V50/TechSpecs / PDI_19_ Pediatric_Safety_for_Selected_Indicators.pdf.

5. GriffinFA, Resar RK. IHIGlobal TriggerTool for Measuring

Tabla 1. Variables asociadas a eventos adversos

\begin{tabular}{lccc}
\hline Variable & OR $_{\mathrm{AI}}$ & IC 95 \% & Valor de $\boldsymbol{p}$ \\
\hline Pacientes con medicamentos de alto riesgo & 2,13 & $1,24-5,12$ & 0,004 \\
Estancia superior a 5 días & 1,84 & $0,11-2,24$ & 0,05 \\
Más de una transferencia asistencial & 2,06 & $1,54-6,76$ & 0,04 \\
Peso & 2,89 & $1,61-4,26$ & 0,01 \\
Asistencia respiratoria mecánica & 0,55 & $0,41-1,26$ & $0,4(N S)$ \\
Sexo femenino & 1,78 & $1,24-2,49$ & 0,01 \\
\hline
\end{tabular}

$\mathrm{OR}_{\mathrm{AJ}}$ : odds ratio ajustado; IC $95 \%$ : intervalo de confianza del $95 \%$; NS: no significativa. 
Adverse Events. 2nd ed. IHI Innovation Series white paper. Cambridge, MA: Institute for Healthcare Improvement; 2009.

6. Classen DC, Resar R, Griffin F, Federico F, et al. Global Trigger Tool shows that adverse events in hospitals may be ten times greater than previously measured. Health Aff (Millwood). 2011; 30(4):581-9.

7. NHS Institute for Innovation and Improvement. Paediatric Trigger. User guide. [Consulta: 3 de diciembre de 2017]. Disponible en: https: / / bmjopen.bmj.com/content / suppl/2014/07/03/bmjopen-2014-005066.DC1/bmjopen2014-005066supp2.pdf

8. Kirkendall ES, Kloppenborg E, Papp J, White D, et al. Measuring adverse events and levels of harm in pediatric inpatients with the Global Trigger Tool. Pediatrics. 2012; 130(5):e1206-14.

9. Davenport MC, Domínguez PA, Ferreira JP, Kannemann $\mathrm{AL}$, et al. Detección de eventos adversos en pacientes pediátricos hospitalizados mediante la herramienta de disparadores globales Global Trigger Tool. Arch Arg Pediatr. 2017; 115(4):357-63.

10. Nacional Coordinating Council for Medication Error Prevention. Taxonomy of Medication Error Now Available. [Consulta: agosto de 2016]. Disponible en: http:/ / www. nccmerp.org/taxonomy-medication-errors-now-available.

11. Institute for Safe Medication Practices. High alert medication lists. [Consulta: agosto de 2016]. Disponibleen: https: / / www.ismp.org/tools/highalertmedicationlists. asp.

12. Stockwell DC, Bisarya H, Classen D, Kirkendall ES, et al. A trigger tool to detect harm in pediatric inpatient settings. Pediatrics. 2015; 135(6):1036-42.

13. Takata GS, Mason W, Taketomo C, Logsdon T, et al. Development, testing, and findings of a pediatric-focused trigger tool to identify medication-related harm in US children's hospitals. Pediatrics. 2008; 121(4):e927-35.

14. Stroupe LM, Patra KP, Dai Z, Lancaster J, et al. Measuring Harm in Hospitalized Children via a Trigger Tool. J Pediatr Nurs. 2017; S0882-5963(16)30295-0.

15. Landrigan CP, Stockwell D, Toomey SL, Loren S, et al. Performance of the Global Assessment of Pediatric Patient Safety (GAPPS) Tool. Pediatrics. 2016; 137(6):e20154076.

16. Palacios-Barahona AU, Bareño-Silva J. Factores asociados a eventos adversos en pacientes hospitalizados en una entidad de salud en Colombia. Rev CES Med.2012;26(1):1928.

17. Kaushal R, Bates DW, Franz C, Soukup JR, et al. Costs of adverse events in intensive care units. Crit Care Med. 2007; 35(11):2479-83.

18. Hug BL, Keohane C, Seger DL, Yoon C, et al. The costs of adverse drug events in community hospitals. Jt Comm J Qual Patient Saf. 2012; 38(3):120-6. 